\title{
ELEMENTARY PROOF OF A FORMULA OF RAMANUJAN
}

\author{
ROBERT L. LAMPHERE
}

ABSTRACT. In this paper we use only elementary calculus to prove Ramanujan's integral formula

$$
\begin{gathered}
\int_{0}^{\infty} x^{m-1} \frac{(1+a b x)\left(1+a b^{2} x\right) \cdots\left(1+a b^{n} x\right) \cdots}{(1+x)(1+b x)\left(1+b^{2} x\right) \cdots\left(1+b^{n} x\right) \cdots} d x \\
=\frac{\pi}{\operatorname{Sin}(m \pi)} \prod_{k=1}^{\infty} \frac{\left(1-b^{k-m}\right)\left(1-a b^{k}\right)}{\left(1-b^{k}\right)\left(1-a b^{k-m}\right)},
\end{gathered}
$$

where $m, a$ and $b$ are positive with $b<1$ and $a<b^{m-1}$.

1. Introduction. In Ramanujan's collected papers [5, p. 57], the following elegant formula is found:

$$
\begin{gathered}
\int_{0}^{\infty} x^{m-1} \frac{(1+a b x)\left(1+a b^{2} x\right) \cdots\left(1+a b^{n} x\right) \cdots}{(1+x)(1+b x)\left(1+b^{2} x\right) \cdots\left(1+b^{n} x\right) \cdots} d x \\
=\frac{\pi}{\operatorname{Sin}(m \pi)} \prod_{k=1}^{\infty} \frac{\left(1-b^{k-m}\right)\left(1-a b^{k}\right)}{\left(1-b^{k}\right)\left(1-a b^{k-m}\right)}
\end{gathered}
$$

where $m, a$ and $b$ are positive with $b<1$ and $0<a<b^{m-1}$.

Ramanujan did not prove his formula. G. H. Hardy [3], using complex variable theory, gave the first proof of it in 1915. And more recently, another evaluation of (1) has been given by R. A. Askey [1]. The purpose of this paper is to give another proof of Ramanujan's formula, but one which does not use any mathematics higher than calculus.

In our proof of (1) we shall assume that $m$ is not an integer and $a$ is not an integral power of $b$. The formulas of these exceptional cases may be found in Ramanujan's paper and are readily deduced from (1).

2. Proof that Ramanujan's integral converges. If we write

$$
R(u)=\int_{0}^{u} x^{m-1} \frac{B(x)}{1+x} d x, \text { where } B(x)=\prod_{k=1}^{\infty} \frac{1+a b^{k} x}{1+b^{k} x},
$$

then, to prove convergence, we need only show that there is a constant $A$, such that $R(u)<A$ for all values of $u$ greater than 0 [4, p. 358].

Received by the editors May 13, 1983.

1980 Mathematics Subject Classification. Primary 26A06. 
Without loss of generality, we assume that $u>1$. Let

$$
T_{m}(u)=\int_{1}^{u} x^{m-1} \frac{B(x)}{1+x} .
$$

Then, by putting $x^{m-1}=x^{m-2}(1+x-1)$, we obtain

$$
T_{m}(u)=\int_{1}^{u} x^{m-2} B(x) d x-T_{m-1}(u) .
$$

Now, changing $b x$ to $x$, we have

$$
\begin{aligned}
\int_{1}^{u} x^{m-2} B(x) d x & =b^{1-m} \int_{b}^{b u} x^{m-2} \frac{1+a x}{1+x} B(x) d x \\
& =b^{1-m} \int_{b}^{b u} x^{m-2} \frac{B(x)}{1+x} d x+a b^{1-m} \int_{b}^{b u} x^{m-1} \frac{B(x)}{1+x} d x .
\end{aligned}
$$

Let $p=[m]$, in which $[m]$ means the greatest integer $\leqslant m$. Then since $\left(1+a b^{i} x\right) /\left(1+b^{p-1+i} x\right)<1$, for all positive integers $i$, it follows that

$$
\frac{B(x)}{1+x}<\frac{1}{1+x} \prod_{k=1}^{p-1} \frac{1}{1+b^{k} x}<x^{-p} b^{-p(p-1) / 2},
$$

and hence

$$
\int_{b}^{b u} x^{m-2} \frac{B(x)}{1+x} d x<\frac{b^{m-1-p(p+1) / 2}}{p+1-m} .
$$

And since

$$
\int_{b}^{b u} x^{m-1} \frac{B(x)}{1+x} d x<R(u)
$$

it follows that

$$
\int_{1}^{u} x^{m-2} B(x) d x<\frac{b^{-p(p+1) / 2}}{p+1-m}+a b^{1-m} R(u) .
$$

Noting that $T_{m-1}(u)>0$, we have, by (3) and (5),

$$
T_{m}(u)<\int_{1}^{u} x^{m-2} B(x) d x<\frac{b^{-p(p+1) / 2}}{p+1-m}+a b^{1-m} R(u) .
$$

Now, from the definition of $R(u), R(u)=R(1)+T_{m}(u)$. Thus,

$$
R(u)<R(1)+\frac{b^{-p(p+1) / 2}}{p+1-m}+a b^{1-m} R(u),
$$

or $R(u)<A$, where

$$
A=\frac{1}{1-a b^{1-m}}\left(R(1)+\frac{b^{-p(p+1) / 2}}{p+1-m}\right) \text { and } u>1 .
$$

Hence, since $R(u) \leqslant R(1)$ when $0<u \leqslant 1$, we have $R(u)<A$ for all $u>0$.

3. Evaluation of $I_{r}$. We require in our demonstration of (1) the value of

$$
I_{r}=\int_{0}^{\infty} x^{m-1} \frac{D(x, r)}{1+x} d x, \quad \text { where } D(x, r)=\prod_{k=1}^{r} \frac{1}{1+b^{k} x},
$$


and $r>p=[m]$. By changing the independent variable $x$ to $b x$, we have

$$
I_{r}=b^{m} \int_{0}^{\infty} x^{m-1}(1+x) \frac{D(x, r+1)}{1+x} d x
$$

Next, using the identity $1+x=1+\left(\left(1+b^{r+1} x\right)-1\right) / b^{r+1}$, we obtain

$$
I_{r}=b^{m-r-1} I_{r}+b^{m-r-1}\left(b^{r+1}-1\right) I_{r+1} \text {. }
$$

Thus, $I_{r+1}=\left(1-b^{r+1-m}\right) I_{r} /\left(1-b^{r+1}\right)$.

Iteration of our last equation gives us

$$
I_{r}=I_{p} \prod_{k=1+p}^{r} \frac{1-b^{k-m}}{1-b^{k}} .
$$

But, our reduction formula cannot be used when $r<p$, since the integrals $I_{p-1}$, $I_{p-2}$, etc. are divergent. We must now evaluate $I_{p}$.

Let $L_{r}=\int_{0}^{\infty} x^{\alpha-1} F_{r}(x) d x$, where $\alpha=m-p, 0 \leqslant r \leqslant p$, and

$$
F_{r}(x)=x^{r} \prod_{k=0}^{r} \frac{1}{1+b^{k}} x \text {. }
$$

Then observe that $L_{p}=I_{p}$.

Now, replace $x$ by $b x$ to obtain

$$
L_{p}=b^{p+\alpha} \int_{0}^{\infty} F_{p}(x) \frac{1+x}{1+b^{p+1} x} x^{\alpha-1} d x .
$$

Then changing $p$ to $p-j$, where $j$ is a positive integer $<p$, we hve

$$
L_{p-j}=b^{m-j} \int_{0}^{\infty} F_{p-j}(x) \frac{1+x}{1+b^{p-j+1} x} x^{\alpha-1} d x .
$$

Thus,

$$
b^{j-m} L_{p-j}-L_{p-j+1}=\int_{0}^{\infty} \frac{F_{p-j}(x)}{1+b^{p-j+1} x} x^{\alpha-1} d x .
$$

But, $L_{p}$ can also be rewritten as

$$
L_{p}=\int_{0}^{\infty} F_{p-1}(x) \frac{x}{1+b^{p} x} x^{\alpha-1} d x,
$$

and therefore, replacing $p$ by $p-j+1$, multiplying both sides of the equation by $b^{p+1-j}$, and subtracting the result from $L_{p-j}$, we have

$$
L_{p-j}-b^{p-j+1} L_{p-j+1}=\int_{0}^{\infty} \frac{F_{p-j}(x)}{1+b^{p-j+1} x} x^{\alpha-1} d x .
$$

Next, eliminating the common integral from (8) and (9) yields the recurrence relation

$$
L_{p-j+1}=-\frac{1-b^{j-m}}{1-b^{p-j+1}} L_{p-j}
$$

from which we get

$$
L_{p}=(-1)^{p} \prod_{k=1}^{p} \frac{1-b^{k-m}}{1-b^{k}} \int_{0}^{\infty} \frac{x^{\alpha-1}}{1+x} d x=\frac{\pi}{\operatorname{Sin} m \pi} \prod_{k=1}^{p} \frac{1-b^{k-m}}{1-b^{k}},
$$


since [2, p. 61]

$$
\int_{0}^{\infty} \frac{x^{\alpha-1}}{1+x} d x=\frac{\pi}{\operatorname{Sin} \alpha \pi}=(-1)^{p} \frac{\pi}{\operatorname{Sin} m \pi}
$$

Hence, collecting our results, we finally have

$$
I_{r}=\frac{\pi}{\operatorname{Sin} m \pi} \prod_{k=1}^{r} \frac{1-b^{k-m}}{1-b^{k}},
$$

for all integers $r>0$.

4. Proof of Ramanujan's formula. Let $I(a, b)$ denote the integral in (1). Then, replacing $1+a b x$ by $1-a b+a b(1+x)$ and using the fact that $I(a, b)$ is convergent, we have

$$
I(a, b)=(1-a b) \int_{0}^{\infty} \frac{x^{m-1}}{1+x} \prod_{k=1}^{\infty} \frac{1+a b^{k+1} x}{1+b^{k} x}+a b \int_{0}^{\infty} x^{m-1} \prod_{k=1}^{\infty} \frac{1+a b^{k+1} x}{1+b^{k} x} d x
$$

or, by changing $b x$ to $x$ in the second integral

$$
I(a, b)=(1-a b) I(a b, b)+a b^{1-m} I(a, b),
$$

and hence

$$
I(a b, b)=\frac{1-a b^{1-m}}{1-a b} I(a, b) .
$$

Thus,

$$
I\left(a b^{r}, b\right)=I(a, b) \prod_{k=1}^{r} \frac{1-a b^{k-m}}{1-a b^{k}},
$$

and on rewriting (11), we have

$$
\begin{aligned}
I(a, b)= & \frac{\pi}{\operatorname{Sin} m \pi}\left\{\prod_{k=1}^{r} \frac{\left(1-b^{k-m}\right)\left(1-a b^{k}\right)}{\left(1-b^{k}\right)\left(1-a b^{k-m}\right)}\right\} \\
& \times \frac{I\left(a b^{r}, b\right)}{(\pi / \operatorname{Sin} \pi m) \prod_{k=1}^{r}\left(\left(1-b^{k-m}\right) /\left(1-b^{k}\right)\right)} .
\end{aligned}
$$

Therefore, if we can show

$$
\operatorname{Limit}_{r \rightarrow \infty} \frac{I\left(a b^{r}, b\right)}{(\pi / \operatorname{Sin} m \pi) \prod_{k=1}^{r}\left(\left(1-b^{k-m}\right) /\left(1-b^{k}\right)\right)}=1,
$$

then the proof of Ramanujan's formula will be complete.

In order to show that the limit in (12) is 1 , we need the following two inequalities:

$$
\begin{aligned}
& \frac{1}{(1+x)(1+b x)\left(1+b^{2} x\right) \cdots\left(1+b^{r-2} x\right)} \\
& >\frac{\left(1+a b^{r} x\right)\left(1+a b^{r+1} x\right) \cdots\left(1+a b^{r+n+1} x\right)}{(1+x)(1+b x)\left(1+b^{2} x\right) \cdots\left(1+b^{r+n} x\right)}
\end{aligned}
$$


and

$$
\begin{aligned}
& \frac{\left(1+a b^{r} x\right)\left(1+a b^{r+1} x\right) \cdots\left(1+a b^{r+n} x\right)}{(1+x)(1+b x)\left(1+b^{2} x\right) \cdots\left(1+b^{r+n+s} x\right)} \\
& >\frac{1}{(1+x)(1+b x)\left(1+b^{2} x\right) \cdots\left(1+b^{r+s-1} x\right)}
\end{aligned}
$$

where $n, r$ and $s$ are positive integers, with $r>p=[m]$ and $0<b^{s}<a<b^{-1}$, and with $n$ independent of both $r$ and $s$.

These inequalities can be easily verified; to establish the first inequality, replace $a$ with $b^{-1}$ and use the fact that $a<b^{-1}$; and to establish the second, replace $a$ with $b^{s}$ and recall $b^{s}<a$.

Now, multiply the above inequalities by $x^{m-1}$ and let $n \rightarrow \infty$; then integrating yields the inequality $I_{r-2} \geqslant I\left(a b^{r-1}, b\right) \geqslant I_{r+s-1}$. From equation (10), we have

$$
\frac{\pi}{\operatorname{Sin} m \pi} \prod_{k=1}^{r-2} \frac{1-b^{k-m}}{1-b^{k}} \geqslant I\left(a b^{r-1}, b\right) \geqslant \frac{\pi}{\operatorname{Sin} m \pi} \prod_{k=1}^{r+s-1} \frac{1-b^{k-m}}{1-b^{k}} .
$$

Letting $r$ tend to $\infty$, we readily deduce (12), and so the proof of (1) is complete.

\section{REFERENCES}

1. R. A. Askey, Ramanujan's extensions of the gamma and beta functions, Amer. Math. Monthly 87 (1980), 346-359.

2. Joseph Edwards, Treatise on integral calculus, vol. 2, reprinted Chelsea, New York, 1922.

3. G. H. Hardy, Proof of a formula of Mr. Ramanujan, Messenger of Math. 44 (1915), 18-21.

4. __ Pure mathematics, 1952; reprinted Cambridge University Press, London, 1963.

5. S. Ramanujan, Collected papers, Chelsea, New York, 1962.

Department of Mathematics, Elmhurst College, Elmhurst, Illinois 60126 